\title{
Two-parameter generalization of the logarithm and exponential functions and Boltzmann-Gibbs-Shannon entropy
}

\author{
Veit Schwämmle and Constantino Tsallis \\ Centro Brasileiro de Pesquisas Fisicas \\ Rua Xavier Sigaud 150, 22290-180 Rio de Janeiro-RJ, Brazil \\ E-mail: veit@cbpf.br, tsallis@cbpf.br
}

\begin{abstract}
The $q$-sum $x \oplus_{q} y \equiv x+y+(1-q) x y\left(x \oplus_{1} y=x+y\right)$ and the $q$ product $x \otimes_{q} y \equiv\left[x^{1-q}+y^{1-q}-1\right]^{\frac{1}{1-q}}\left(x \otimes_{1} y=x y\right)$ emerge naturally within nonextensive statistical mechanics. We show here how they lead to two-parameter (namely, $q$ and $q^{\prime}$ ) generalizations of the logarithmic and exponential functions (noted respectively $\ln _{q, q^{\prime}} x$ and $e_{q, q^{\prime}}^{x}$, as well as of the Boltzmann-Gibbs-Shannon entropy $S_{B G S} \equiv-k \sum_{i=1}^{W} p_{i} \ln p_{i}$ (noted $S_{q, q^{\prime}}$ ). The remarkable properties of the $\left(q, q^{\prime}\right)$ generalized logarithmic function make the entropic form $S_{q, q^{\prime}} \equiv k \sum_{i=1}^{W} p_{i} \ln _{q, q^{\prime}}\left(1 / p_{i}\right)$ to satisfy, for large regions of $\left(q, q^{\prime}\right)$, important properties such as expansibility, concavity and Lesche-stability, but not necessarily composability.
\end{abstract}

PACS numbers: 02.10.-v, 02.70.Rr, 05.90.+m

\section{INTRODUCTION}

Boltzmann-Gibbs $(B G)$ statistical mechanics provides a powerful tool for understanding how fast microscopic physics with short-range interactions affects the thermodynamics of a system on much larger space-time scales. It is based on the (Boltzmann-Gibbs or Boltzmann-Gibbs-Shannon) entropy

$$
S_{B G S} \equiv-k \sum_{i=1}^{W} p_{i} \ln p_{i}=k \sum_{i=1}^{W} p_{i} \ln \frac{1}{p_{i}}\left(\sum_{i=1}^{W} p_{i}=1\right),
$$

hence, if $p_{i}=1 / W(\forall i), S_{B G S}=k \ln W$.

However, the $B G$ theory turns out to be inadequate for various complex natural, artificial and social systems. Such is, for instance, the case where a zero maximal Lyapunov exponent is present (e.g., long-range-interaction many-body Hamiltonian systems, nonlinear dynamical systems at the edge of chaos, and others). Typically, such situations are dominated by power-laws instead of exponential distributions [1, 2]. In order to deal with at least part of such systems, it was proposed in 1988 [3], a 
generalization of the $B G$ theory, referred to as nonextensive statistical mechanics [4, 5]. It is based on the entropy

$$
S_{q} \equiv k \frac{1-\sum_{i=1}^{W} p_{i}^{q}}{q-1}=k \sum_{i=1}^{W} p_{i} \ln _{q}\left(\frac{1}{p_{i}}\right) \quad\left(q \in \mathcal{R}, \sum_{i=1}^{W} p_{i}=1\right),
$$

(hence, if $\left.p_{i}=1 / W(\forall i), S_{q}=k \ln _{q} W\right)$ where

$$
\ln _{q} x \equiv \frac{x^{1-q}-1}{1-q}\left(\ln _{1} x=\ln x\right) .
$$

This entropy succeeds to widen the range of applicability of statistical mechanical concepts to many systems. It fulfills thermodynamically relevant properties such as expansibility, composability, Lesche-stability and concavity (for $q>0$ ). The roles played in the traditional theory by the logarithmic and exponential functions are played, in the generalized theory, by the so-called $q$-logarithm, as given by Eq. (3), and its inverse, the $q$-exponential [6]:

$$
e_{q}^{x} \equiv[1+(1-q) x]_{+}^{\frac{1}{1-q}} \quad\left(e_{1}^{x}=e^{x}\right),
$$

where $[\ldots]+$ vanishes if its argument is nonpositive. This naturally led to the construction of a family of functions based on the $q$-generalized logarithm and its inverse function [7].

Consistently, nonlinear generalized algebraic forms emerge (see also [8]), namely the $q$-sum [9] and the $q$-product [9, 10] respectively defined as follows:

$$
x \oplus_{q} y \equiv x+y+(1-q) x y \quad\left(x \oplus_{1} y=x+y\right),
$$

and

$$
x \otimes_{q} y \equiv\left(x^{1-q}+y^{1-q}-1\right)^{\frac{1}{1-q}} \quad\left(x \otimes_{1} y=x y\right) .
$$

Additionally, with these mathematical generalizations of the sum and the product, a new algebra could be constructed which appears to have some peculiar properties not found for other algebras [9, 10]. The $q$-product and the $q$-sum maintain the structure given by Eq. (7) by converting the sum and the product into these non-linear operators.

We can straightforwardly verify the following properties:

$$
\begin{aligned}
& \ln (x \cdot y)=\ln x+\ln y \\
& \ln _{q}(x \cdot y)=\ln _{q} x \oplus_{q} \ln _{q} y \\
& \ln _{q}\left(x \otimes_{q} y\right)=\ln _{q} x+\ln _{q} y
\end{aligned}
$$

In the following section (Sec. 2) we show that an uniquely further generalized logarithmic function, denoted $\ln _{q, q^{\prime}} x$, exists which enables the unification of Eqs. (77.9) as follows:

$$
\ln _{q, q^{\prime}}\left(x \otimes_{q} y\right)=\ln _{q, q^{\prime}} x \oplus_{q^{\prime}} \ln _{q, q^{\prime}} y .
$$

In Sec. 3 we present some general properties of this function. In Sec. 4 we introduce a two-parameter generalization of the $B G S$ entropy, and discuss various of its properties. We finally conclude in Sec. 5 . 


\section{DERIVATION OF THE TWO-PARAMETER GENERALIZATION OF THE LOGARITHMIC FUNCTION}

Eq. (10) constitutes a strongly nonlinear functional equation. A general analytic expression of $\ln _{q, q^{\prime}} x$ needs to satisfy the limiting cases $\ln _{q, 1} x=\ln _{1, q} x=\ln _{q} x$.

In order to simplify Eq. (10), we set $x=y$ and, inspired by the expression on the left hand side of Eq. (9), write $g(z)=g\left(\ln _{q} x\right)=\ln _{q, q^{\prime}} x$. By inserting $g(z)$ in Eq. (10), this functional equation transforms into the much simpler form

$$
g(2 z)=2 g(z)+\left(1-q^{\prime}\right)[g(z)]^{2} .
$$

We then verify that the ansatz $g(z)=\frac{1}{1-q^{\prime}}\left(b^{z}-1\right)$ solves Eq. (11) exactly, thus leading to,

$$
\ln _{q, q^{\prime}} x=\frac{1}{1-q^{\prime}}\left(b^{\frac{1}{1-q}\left(x^{1-q}-1\right)}-1\right),
$$

where $b$ corresponds to a constant that still needs to be determined. It is important to point out that $\ln _{q, q^{\prime}}(x)$ solves Eq. (10) also for the more general case $x \neq y$.

In order to determine $b$, we focus on the point $\ln _{q, q^{\prime}} 1=0$, which is expected to hold for all $b$ and all $\left(q, q^{\prime}\right)$, analogously to the fact that $\ln _{q} 1=0$ for all $q$. We assume also that, at this point, the entire family $\ln _{q, q^{\prime}} x$ has one and the same slope, i.e., $\left.\frac{d}{d x} \ln _{q, q^{\prime}} x\right|_{x=1}=1$, once again in full analogy with $\ln _{q} x$. Then, we verify that our new function, Eq. (12), takes the following more specific form:

$$
\ln _{q, q^{\prime}} x=\frac{1}{1-q^{\prime}}\left[\exp \left(\frac{1-q^{\prime}}{1-q}\left(x^{1-q}-1\right)\right)-1\right] .
$$

Indeed, we can prove that $\ln _{q, q^{\prime}} x$ provides a generalized function of the $q$-logarithm with $b=\exp \left(1-q^{\prime}\right)$ by setting either $q$ or $q^{\prime}$ equal to unity. Eq. (13) can be rewritten as follows:

$$
\ln _{q, q^{\prime}} x=\ln _{q^{\prime}} e^{\ln _{q} x} .
$$

The relations

$$
\ln _{q, 1} x=\ln _{1, q} x=\ln _{q} x
$$

are easily recovered by evaluating Eq. (13) in the limits $q \rightarrow 1$ and $q^{\prime} \rightarrow 1$.

In Fig. 1 w wllustrate the two-parameter generalized logarithmic function for typical $q=q^{\prime}$ cases. Typical $q \neq q^{\prime}$ cases are illustrated in Fig. 2 ,

\section{PROPERTIES}

In this section we present some general properties of the two-parameter generalized logarithm, some of which will be useful in the discussion of Sec. 4.

As can be obtained from Eq. (13) (and observed in Figs. 1 and 24), $\ln _{q, q^{\prime}} x$ monotonically increases with $x$. Thus, an unique inverse function of $\ln _{q, q^{\prime}} x$, namely 
the two-parameter generalized exponential function, exists for arbitrary values of $q$ and $q^{\prime}$ :

$$
\mathrm{e}_{q, q^{\prime}}^{y}=\left\{1+\frac{1-q}{1-q^{\prime}} \ln \left[1+\left(1-q^{\prime}\right) y\right]\right\}^{\frac{1}{1-q}}=e_{q}^{\ln e_{{q^{\prime}}^{\prime}}} .
$$

There are two cut-offs, namely for $y\left(1-q^{\prime}\right)>1$ and for $\{\ldots\}<0$. This can be easily understood looking at the shape of the generalized logarithm in Figs. 1 and $2, \ln _{q, q^{\prime}} x$ saturates for $x \rightarrow \infty$ if at least one of the parameters is greater than unity (either $q>1$ or $q^{\prime}>1$ ). Moreover, it also approaches a finite value for $x \rightarrow 0$ if at least one of the parameters is less than unity (either $q<1$ or $q^{\prime}<1$ ).

It is remarkable that the functional form of the present two-parameter generalization of the logarithmic function contains the standard exponential function, $\mathrm{e}^{x}$. The reciprocal happens with the functional form of its inverse function, $\exp _{q, q^{\prime}}(x)$, which contains $\ln x$.

Many functions, e.g. $\sin x$ and $\cos x$, can be expressed in terms of the exponential function. Similarly, it should be possible to construct a new family of functions using Eqs. (13) and (16).

Let us calculate here the first derivative of $\ln _{q, q^{\prime}} x$ :

$$
\frac{d \ln _{q, q^{\prime}} x}{d x}=x^{-q} \exp \left(\frac{1-q^{\prime}}{1-q}\left(x^{1-q}-1\right)\right) .
$$

The slope of $\ln _{q, q^{\prime}} x$ remains positive for all $x>0$, which is consistent with the already mentioned monotonicity of the generalized logarithm for all values of $\left(q, q^{\prime}\right)$.

The shape of $\ln _{q, q^{\prime}} x$ in Figs. 1 and 2 suggests that there may exist some symmetry with respect to the point $(x, y)=(1,0)$. Indeed, the transformation $q \rightarrow 2-q$, well known to be an important relation in nonextensive thermostatistics [4], makes $\ln _{q, q^{\prime}} x$ to satisfy the following property:

$$
\ln _{q, q^{\prime}} \frac{1}{x}=-\ln _{2-q, 2-q^{\prime}} x \text {. }
$$

\section{TWO-PARAMETER GENERALIZATION OF THE BOLTZMANN-GIBBS-SHANNON ENTROPY}

We construct here an entropic functional based on the two-parameter generalization of the standard logarithm.

Along the lines of Eqs. (1) and (2) we can construct a new entropic functional as follows:

$$
S_{q, q^{\prime}} \equiv k \sum_{i=1}^{W} p_{i} \ln _{q, q^{\prime}} \frac{1}{p_{i}}=\frac{k}{1-q^{\prime}} \sum_{n=1}^{W} p_{i}\left(e^{\frac{1-q^{\prime}}{1-q}\left(p_{i}^{q-1}-1\right)}-1\right)
$$

(with $\sum_{i=1}^{W} p_{i}=1$ ) hence, if $p_{i}=1 / W(\forall i), S_{q, q^{\prime}}=k \ln _{q, q^{\prime}} W$.

Lesche-stability (or experimental robustness): The functional form of $\ln _{q, q^{\prime}} x$ being analytic in $x$, this entropy is Lesche-stable (experimentally robust) [11]. 

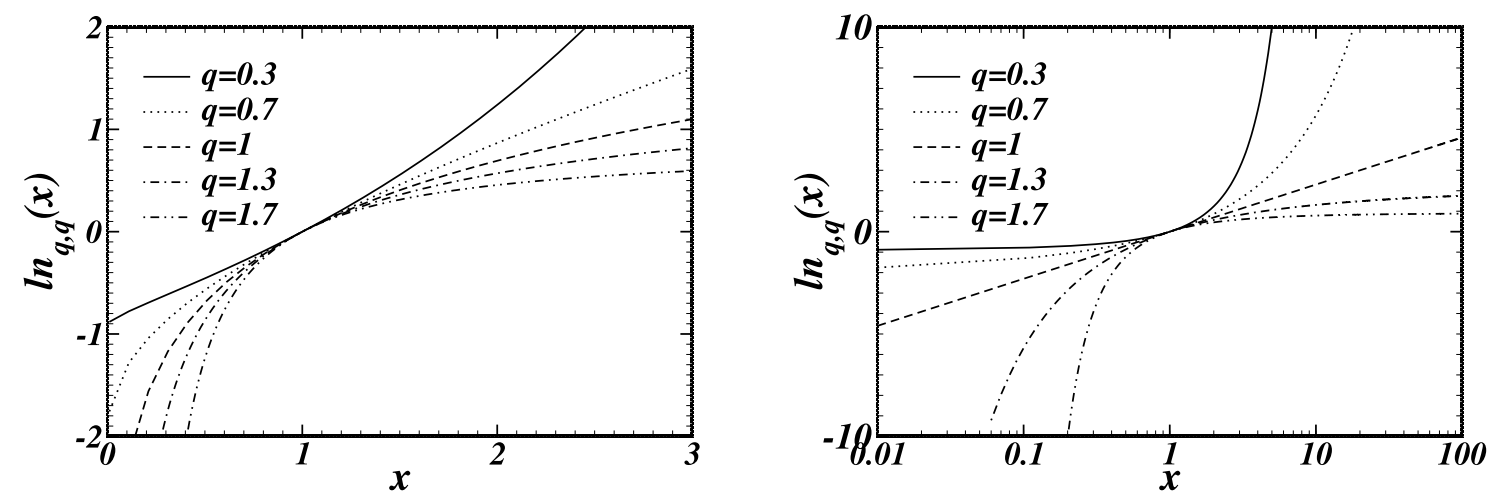

Figure 1. Illustration of the generalized logarithm, Eq. (13), setting $q=q^{\prime}$, in linear scales (left) and semi-logarithmic scales (right).

Expansibility: A zero-probability $\left(p_{i}=0\right)$ state does not contribute to the entropy if at least one of the parameters is greater than unity $\left(q>1\right.$ or $\left.q^{\prime}>1\right)$. Note that $q>0$ $\left(q^{\prime}>0\right)$ in the limit $q^{\prime}=1(q=1)$. Therefore $S_{q, q^{\prime}}\left(p_{1}, p_{2}, \ldots, p_{W}, 0\right)=S_{q, q^{\prime}}\left(p_{1}, p_{2}, \ldots, p_{W}\right)$, i.e., $S_{q, q^{\prime}}$ is expansible within the above given limits for the two parameters.

Concavity: An important requirement for an entropic function is that its curvature does not change with $\left\{p_{i}\right\}$ (sufficient although not necessary condition for being concave or convex, i.e., an entropy can still have a fixed sign of its curvature for parameter values not satisfying this condition). Figs. 3A-D illustrate $S_{q, q^{\prime}}$ for a system of two states with probabilities $p$ and $(1-p)$ for typical values of $\left(q, q^{\prime}\right)$. In Fig. 3A (Fig. 3B $)$, the curvature of $S_{q, q^{\prime}}$ is negative (positive) for all $\left(q, q^{\prime}\right)$, and the extremum lies at $p=0.5$ as expected.

In order to determine the regions of the parameter space where the curvature of $S_{q, q^{\prime}}$ does not change, we focus on the curvature of $p_{i} \ln _{q, q^{\prime}}\left(1 / p_{i}\right)$. We check whether there is any change of the sign of the curvature in the interval $0 \leq p_{i} \leq 1$. We verify

$$
\frac{d^{2}}{d p_{i}^{2}} p_{i} \ln _{q, q^{\prime}} \frac{1}{p_{i}}=e^{\frac{1-q^{\prime}}{1-q}\left(p_{i}^{q-1}-1\right)}\left[p_{i}^{2 q-3}\left(1-q^{\prime}\right)-q p_{i}^{q-2}\right] .
$$

Concavity is guaranteed if Eq. (20) remains negative. This is fulfilled if $q+q^{\prime} \geq 1$ in the limit $p_{i}=1$, and $q^{\prime} \geq 1$ if $q<1$ in the limit $p_{i} \rightarrow 0$. Analogously, convexity is guaranteed if $q+q^{\prime} \leq 1$ in the limit $p_{i}=1$, and $q, q^{\prime}<1$ in the limit $p_{i} \rightarrow 0$.

Fig. 4 exhibits the various regions for the curvature with respect to $q$ and $q^{\prime}$. We can see that two regions (III and IV) may have non fixed curvature sign. An analysis of the shape of $S_{q, q^{\prime}}$ with $W=2$ for several values of $q$ and $q^{\prime}$ confirms this but suggests that parts of regions III and IV may still have fixed curvature sign (see also Fig. 3 for examples of $S_{q, q^{\prime}}$ corresponding to these regions).

About composability: Consider a system $A+B$ composed by two probabilistically independent subsystems $A$ and $B$ (i.e., $p_{i, j}^{A+B}=p_{i}^{A} p_{j}^{B}$ ). An entropic functional $S$ is said 

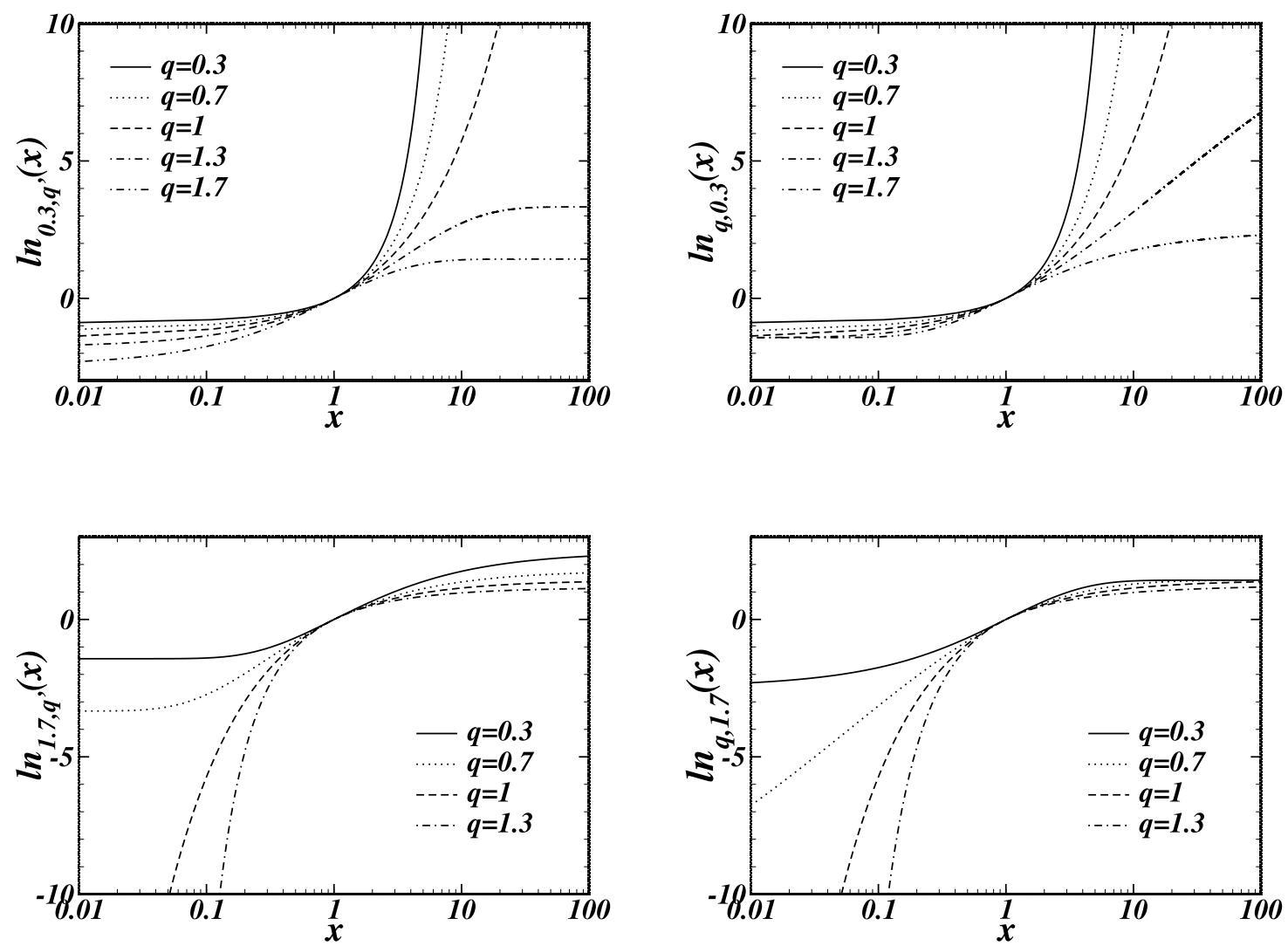

Figure 2. Illustration of the generalized logarithm for typical values of the case $q \neq q^{\prime}$. We set $q=0.3$ and vary $q^{\prime}$ (upper left), then set $q^{\prime}=0.3$ and vary $q$ (upper right). The lower figures correspond to fixed $q=1.7$ (left) and $q^{\prime}=1.7$ (right). Note, that the curves in the left figures and the ones in the right figures can be transformed into each other by applying Eq. (18).

to be composable if its value $S(A+B)$ depends from the probabilities $\left\{p_{i}^{A}\right\}\left\{p_{j}^{B}\right\}$ only through $S(A), S(B)$ and generic indices, i.e., if $S(A+B)=\Phi[S(A), S(B)$, indices $]$, where $\Phi$ is some univalued function. Any additive entropic functional (such as the BGS and the Renyi ones) trivially is composable (e.g., $\left.S_{B G S}(A+B)=S_{B G S}(A)+S_{B G S}(B)\right)$. The nonadditive entropy $S_{q}(q \neq 1)$ also is composable since $\frac{S_{q}^{A+B}}{k}=\frac{S_{q}^{A}}{k} \oplus_{q} \frac{S_{q}^{B}}{k}=\frac{S_{q}(A)}{k}+$ $\frac{S_{q}(B)}{k}+(1-q) \frac{S_{q}(A)}{k} \frac{S_{q}(B)}{k}$. Composability is an important property for thermodynamical applications, not so for applications such as signal or image processing, cybernetics, and others.

Let us now check whether $S_{q, q^{\prime}}$ is composable. We start by considering a microcanonical ensemble, i.e., equal probabilities. We have $S_{q, q^{\prime}}^{A+B}=k \ln _{q, q^{\prime}}\left(W_{A} W_{B}\right), S_{q, q^{\prime}}^{A}=$ $k \ln _{q, q^{\prime}} W_{A}$ and $S_{q, q^{\prime}}^{B}=k \ln _{q, q^{\prime}} W_{B}$. It straightforwardly follows that

$$
\frac{S_{q, q^{\prime}}^{A+B}}{k}=\frac{1}{1-q^{\prime}}\left\{e^{\frac{1-q^{\prime}}{1-q} \ln \left[1+\left(1-q^{\prime}\right) \frac{S_{q, q^{\prime}}^{A}}{k}\right] \ln \left[1+\left(1-q^{\prime}\right) \frac{S_{q, q^{\prime}} B}{k}\right.}\right]
$$



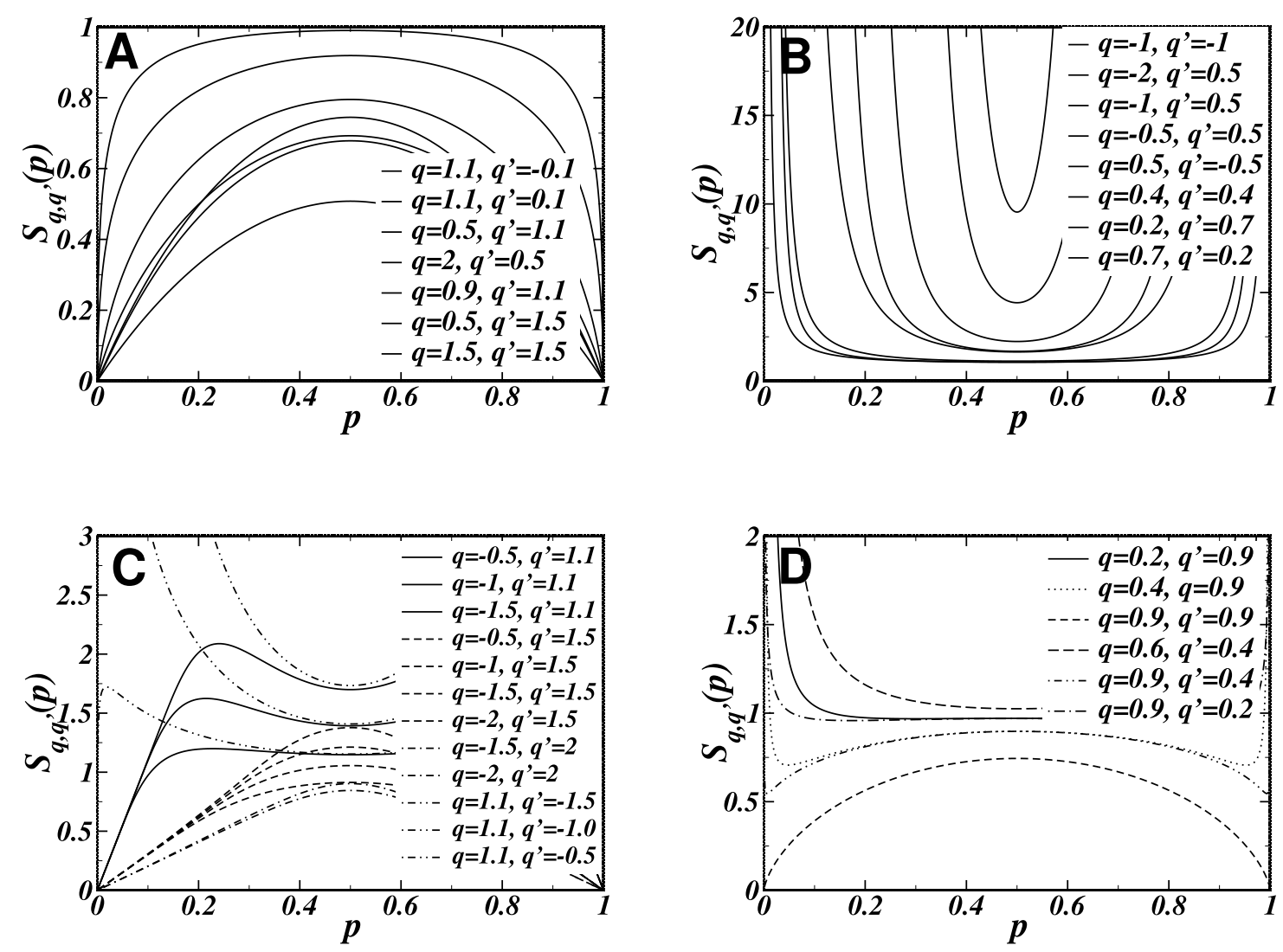

Figure 3. Illustration of the two-state generalized entropy $S_{q, q^{\prime}}$ for typical values of $\left(q, q^{\prime}\right)$. A: concavity (region I in Fig. 4). B: convexity (region II in Fig. (4). C: curvature can change (region III in Fig. 迆. D: curvature can change (region IV in Fig. (4).

$$
\left.\times\left[1+\left(1-q^{\prime}\right) \frac{S_{q, q^{\prime}}^{A}}{k}\right]\left[1+\left(1-q^{\prime}\right) \frac{S_{q, q^{\prime}}^{B}}{k}\right]-1\right\} .
$$

The meaning of such a relation (pseudo-additivity) is uneasy to interpret. Nevertheless, we can present Eq. (21) in a simpler manner by introducing the transformation $Y(S) \equiv \ln \left\{1+\frac{1-q}{1-q^{\prime}} \ln \left[1+\left(1-q^{\prime}\right)(S / k)\right]\right\}$. We then obtain

$$
Y\left(S^{A+B}\right)=Y\left(S^{A}\right)+Y\left(S^{B}\right) .
$$

Composability of the present entropic form $S_{q, q^{\prime}}$ requires that the same relation in Eq. (22) remains valid for other thermostatistical statistics such as, for instance, that of the canonical ensemble. However, our studies indicate that this is not the case for arbitrary distributions $\left\{p_{i}\right\}$. Numerical verifications confirmed this result. Therefore $S_{q, q^{\prime}}$ is in general not composable. 


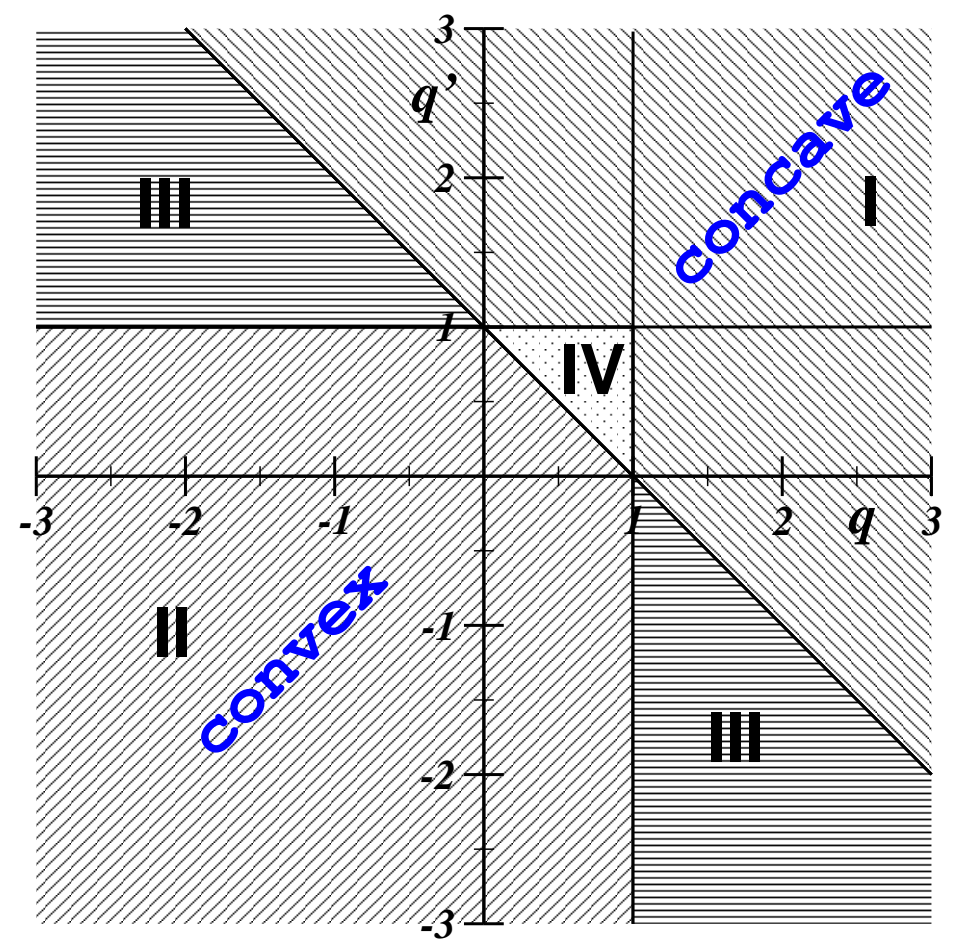

Figure 4. The different regions of the entropy $S_{q, q^{\prime}}$ with respect to its curvature (see also Fig. 31). The point $q=q^{\prime}=1$ corresponds to the Boltzmann-Gibbs-Shannon entropy. The lines $q=1$ and $q^{\prime}=1$ correspond to the nonadditive entropy $S_{q}$. This diagram exhibits $\left(q, q^{\prime}\right) \rightarrow\left(q^{\prime}, q\right)$ symmetry although generically $S_{q, q^{\prime}} \neq S_{q^{\prime}, q}$.

\section{CONCLUSION}

We found an explicit generalized logarithmic function which nicely closes the picture obtained with the $q$-logarithm using the $q$-sum and the $q$-product as basic mathematical operations. It is possible to use the new logarithm, $\ln _{q, q^{\prime}} x$, and its inverse function, $e_{q, q^{\prime}}^{x}$, in order to construct a new family of functions such as $\sin _{q, q^{\prime}} x$ and $\cos _{q, q^{\prime}} x$. It remains to be tested if these new findings can be useful to describe, in some way, some behavior of natural, artificial or social complex systems, or if they can be useful in applied areas such as signal and image processing, cybernetics, optimization algorithms, information theory, among others. Even if they do not immediately exhibit applications, they remain as elegant mathematical constructions. Does $\ln _{q, q^{\prime}} x$ constitute a "supergeneralized" logarithm possibly useful as the basis for a more general entropy? We engaged this question by showing that various important properties (concavity, Leschestability, expansibility) presumably needed for a physical entropy are fulfilled in this case providing that some minimal restrictions are imposed to $\left(q, q^{\prime}\right)$. However, one crucial property failed to be valid in general, namely composability. The fact that this entropy is not composable leads to the assumption that it may be not able to play a thermodynamical role comparable to the nonadditive entropy $S_{q}$ on which nonextensive statistical mechanics is based. 
As a last remark, let us mention that, along the present lines, Renyi' s entropy

$S_{q}^{R} \equiv \frac{\ln \sum_{i=1}^{W} p_{i}^{q}}{1-q}$ can also be (trivially) generalized into a two-parameter one, namely $\frac{\ln _{q^{\prime}} \sum_{i=1}^{W} p_{i}^{q}}{1-q}$.

\section{Acknowledgements}

One of us (C. T.) has benefited from interesting conversations with E.P. Borges, M. Gell-Mann and R. Hersh at the Santa Fe Institute, New Mexico, where this project started. Financial support by Pronex/MCT, CNPq and Faperj (Brazilian Agencies) is gratefully acknowledged as well.

[1] M. Gell-Mann and C. Tsallis, editors. Nonextensive Entropy - Interdisciplinary Applications, New York, 2004. Oxford University Press.

[2] J. P. Boon and C. Tsallis, editors. Nonextensive Statistical Mechanics: New Trends, New Perspectives, volume 36. Europhysics News, 2005.

[3] C. Tsallis. J. Stat. Phys., 52:479, 1988. For a regularly updated bibliography, see http://tsallis.cat.cbpf.br/biblio.htm.

[4] E. M. F. Curado and C. Tsallis. J. Phys. A, L69:24, 1991. Corrigenda: 24 (1991) 3187 and 25 (1992) 1019.

[5] C. Tsallis, R. S. R.S. Mendes, and A. R. Plastino. Physica A, 261:534, 1998.

[6] C. Tsallis. Quimica Nova, 17:468, 1994.

[7] E. P. Borges. J. Phys. A, 31:5281, 1998.

[8] G. Kaniadakis. Phys. Rev. E, 66:056125, 2002.

[9] E. P. Borges. Physica A, 340:95, 2004.

[10] L. Nivanen, A. Le Mehaute, and Q. A. Wang. Rep. Math. Phys., 52:437, 2003.

[11] E. M. F. Curado and F. D. Nobre. Physica A, 335:94, 2004. 